\title{
Effective tranexamic acid concentration for $95 \%$ inhibition of tissue-type plasminogen activator-induced fibrinolysis in full-term pregnant women.
}

\author{
Lechien A, Faraoni D, El Kenz H, Van der Linden P. \\ Dpt Anaesthesia - CHU Brugmann - HUDERF, Université Libre de Bruxelles, Belgium
}

\section{Introduction}

$\checkmark$ Postpartum hemorrhage (PPH) is the leading cause of maternal mortality and morbidity worldwide.

$\checkmark$ Tranexamic acid (TXA) has been shown to reduce blood loss, blood products transfusion requirements and mortality in many types of surgery and polytrauma (1).

$\checkmark$ A recent study reported a reduction of mortality associated with the use of TXA in women presenting PPH (2). However, further studies are needed to define the pharmacokinetic and pharmacodynamic characteristics of TXA in pregnant women in order to define the optimal administration scheme in $\mathrm{PPH}$.

$\checkmark$ The first objective of our prospective ex-vivo study was to define the minimum TXA concentration required to inhibit fibrinolysis in full-term pregnant women.

$\checkmark$ The second was to compare these results to those obtained in healthy non-pregnant volunteers.

\section{Methods}

$\checkmark$ After IEC and written informed consent, blood samples of 30 healthy full-term pregnant women and 10 healthy non-pregnant women were studied on the ROTEM device.

$\checkmark$ In-vitro maximal fibrinolysis was induced by adding a supraphysiologic concentration of tissue-type plasminogen activator (t-PA) to blood samples. Increasing TXA concentrations (0 to 40 $\mathrm{ng} / \mathrm{ml}$ ) were then spiked into the ROTEM test and inhibition of fibrinolysis was assessed using the LI30 parameter of the ROTEM measured on EXTEM and NATEM tests.

$\checkmark$ Effective TXA concentrations required to achieve 95\% inhibition of fibrinolysis (EC95) was extrapolated using nonlinear regression. An extra sum-of-squares $F$ test was used to compare EC95 obtained in pregnant and non-pregnant women.

\section{Results}

$\checkmark$ Pregnant woman presented a higher weight, body mass index, fibrinogen level and lower hemoglobin and platelets level as well as a shorter APTT compared to non-pregnant volunteers.

$\checkmark$ EC95 was statistically higher in pregnant woman compared to non-pregnant volunteers on both EXTEM and NATEM tests.
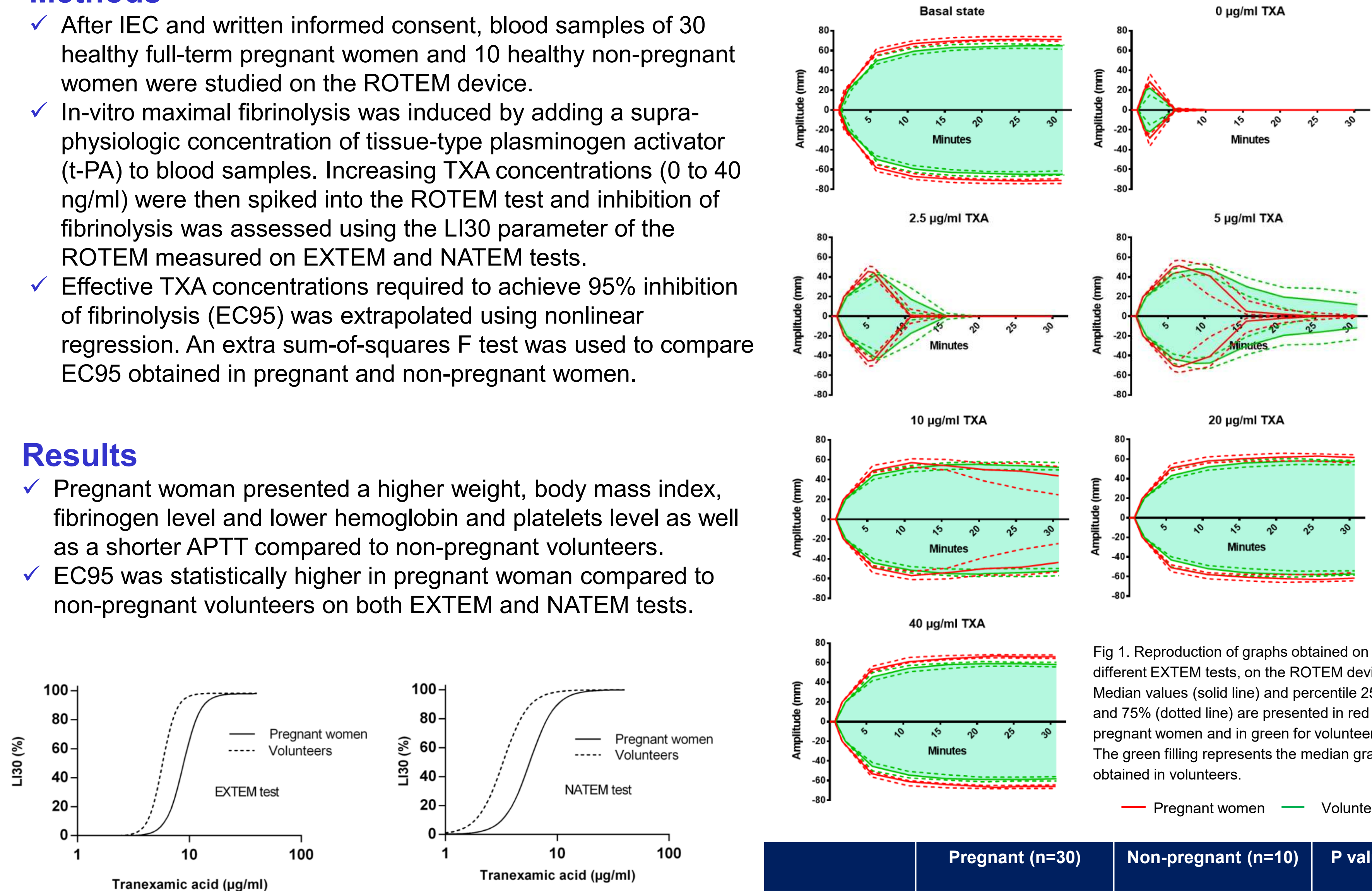

$10 \mu \mathrm{g} / \mathrm{ml}$ TXA
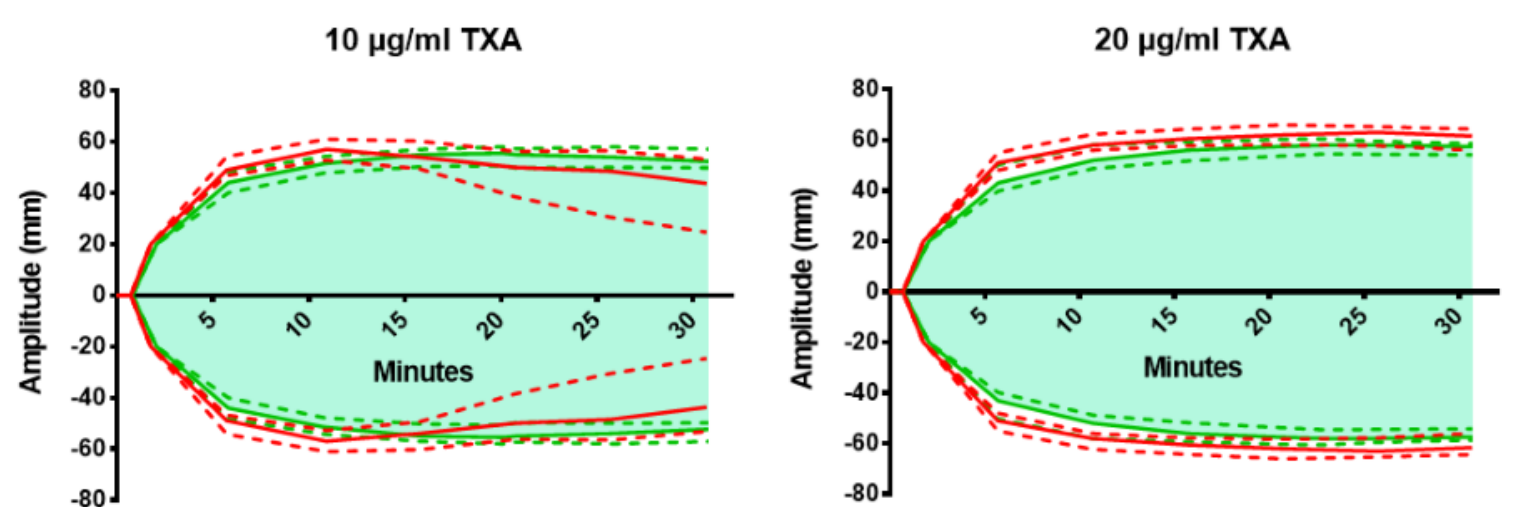

2 and 3. Nonlinear regression for EXTEM test (left) and NATEM test (right) : TXA concentration after logarithmic transformation

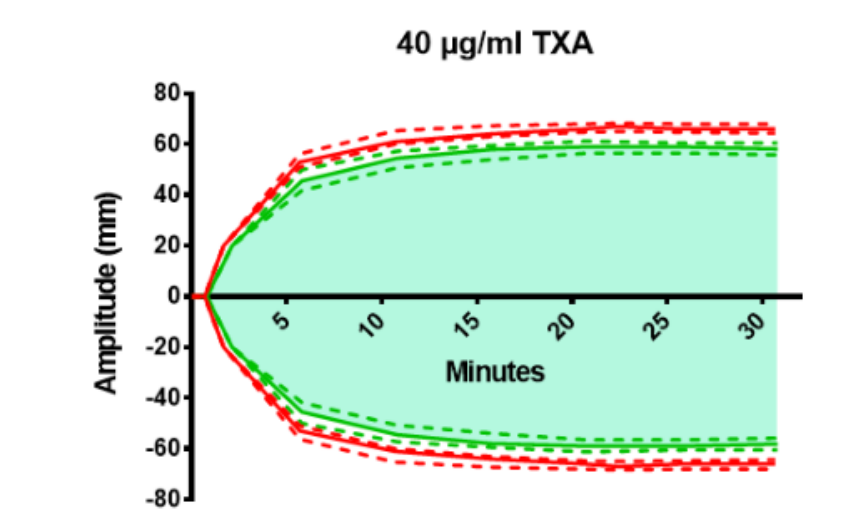

Fig 1. Reproduction of graphs obtained on the different EXTEM tests, on the ROTEM device. Median values (solid line) and percentile $25 \%$ and $75 \%$ (dotted line) are presented in red for pregnant women and in green for volunteers. The green filling represents the median graph obtained in volunteers.

— Pregnant women — Volunteers

\begin{tabular}{|l|c|c|c|}
\hline & Pregnant $(\mathbf{n}=\mathbf{3 0})$ & Non-pregnant $(\mathbf{n = 1 0})$ & P value \\
\hline EC95 (EXTEM) & $14.73(12.35$ to 17.55$)$ & $8.74(5.49$ to 13.92$)$ & $<0.001$ \\
\hline EC95 (NATEM) & $11.19(8.31$ to 15.07$)$ & $6.820(5.30$ to 8.77$)$ & $<0.001$ \\
\hline
\end{tabular}

Values are concentrations and $\mathrm{Cl} 95 \%$, expressed in $\mu \mathrm{g} / \mathrm{ml}$

\section{Conclusions}

$\checkmark$ Despite a decreased fibrinolytic activity associated with a prothrombotic state in the 3rd trimester of pregnancy (3), our results suggest a higher fibrinolytic potential in pregnant women compared to non-pregnant volunteers.

$\checkmark$ The next step should be to determine if the TXA doses used routinely are able to inhibit t-PA-induced fibrinolysis in our ex-vivo model. 